\title{
Bose polaron in spherical trap potentials: Spatial structure and quantum depletion
}

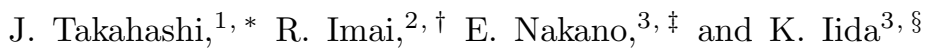 \\ ${ }^{1}$ Department of Electronic and Physical Systems, Waseda University, Tokyo 169-8555, Japan \\ ${ }^{2}$ Department of Materials Science, Waseda University, Tokyo 169-8555, Japan \\ ${ }^{3}$ Department of Mathematics and Physics, Kochi University, Kochi 780-8520, Japan
}

(Dated: June 28, 2019)

\begin{abstract}
We investigate how the presence of a localized impurity in a Bose-Einstein condensate of trapped cold atoms that interact with each other weakly and repulsively affects the profile of the condensed and excited components at zero temperature. By solving the Gross-Pitaevskii and Bogoliubovde Gennes equations, we find that an impurity-boson contact attraction (repulsion) causes both components to change in spatial structure in such a way as to be enhanced (suppressed) around the impurity, while slightly declining (growing) in a far region from the impurity. Such behavior of the quantum depletion of the condensate can be understood by decomposing the impurity-induced change in the profile of the excited component with respect to the radial and azimuthal quantum number. A significant role of the centrifugal potential and the "hole" excitation level is thus clarified.
\end{abstract}

\section{INTRODUCTION}

Polarons are quasiparticles conceptually well established in solid state physics. For instance, a conducting electron in an ionic crystal moves together with the locally induced polarization to form a polaron that has the energy spectrum modified from that of an electron in vacuum 1 13 . Such a medium-modified electron serves as an essential building block for more complex manybody phenomena, e.g., high $T_{c}$ superconductivity [4] . Recently cold atomic gas experiments have offered various types of atomic polarons, i.e., impurities (minority atoms) that are either immersed in a trapped Bose gas with Bose-Einstein condensation (BEC) or in a degenerate Fermi gas and eventually dressed by a virtual cloud of the corresponding particle-hole excitations. The former and latter are called Bose polarons [7-13] and Fermi polarons 14 18, respectively. At sufficiently low temperatures, the interaction between impurity and medium atoms is characterized by a low-energy $s$-wave scattering length, which is in turn tunable by external magnetic fields as predicted by the Fano-Feshbach theory [19]. The polaron energy and spectral strength have been measured by, e.g., radio frequency spectroscopy [11, 12, 14, 15, which utilizes the radio absorption probability of impurity atoms in two different hyperfine states that interact with medium atoms only weakly and relatively strongly.

The above experiments have also energized theoretical investigations, which include various issues of atomic polarons in zero-temperature media: quasiparticle properties of Bose polarons [20 30] and Fermi polarons 3144, the self-localization of impurities in quasi-1D BEC media [45 49], polaronic spectral changes from a weak

\footnotetext{
* takahashi.j@aoni.waseda.jp

$\dagger$ rimai@asagi.waseda.jp

$\ddagger$ e.nakano@kochi-u.ac.jp

$\S$ iida@kochi-u.ac.jp
}

to strong coupling regime for attractive interactions $[50$ 54, few-body physics around the unitarity limit [55 59], dynamics of the polaron formation 59 62, and open quantum dynamics 63 65. Beside these 'conventional' atomic polarons, more exotic ones have been proposed, e.g., $p$-wave 66] and dipolar-type 67, 68, polarons, angulons 69 72, and Bose polarons near the transition temperature [73, 74. Moreover, thermal evolution of Fermi polarons has been recently in the recent experiment 75 . prior to theoretical investigations [76, 77] and critical properties of Bose polarons have been experimentlly observed [78. Whereas most of the theoretical studies mentioned above assume that the system is spatially uniform, Bose polarons in rotationally symmetric trap potentials [79, 80, that are used in experiments have been theoretically studied to figure out the ground-state properties at a given total angular momentum, a conserved quantity in the system considered here. Note that the total momentum is the corresponding quantity in translationallysymmetric uniform systems.

Up to now, however, theoretical studies of Bose polarons have yet to reveal the detailed structures of the condensate and fluctuations in the presence of impurity, especially in 3D trapped systems. This is because the Bogoliubov approximation has been used basically within a theoretical framework that assumes that the presence of the impurity does not change the spatial profile of the condensate nor the number of condensed bosons. The Bogoliubov approximation is supposed to be valid if the fraction of non-condensed bosons that are caused to occur by all interactions assumed in the system is negligibly small $(\ll 1)$. There are some estimates of this condition with respect to the boson-boson and the impurity-boson interaction strengths 81, 82. In general, a repulsive interaction among bosons disturbs them in their condensing into a coherent state by causing fluctuations to generate a finite fraction of non-condensed bosons even at zero temperature, a phenomenon referred to as the quantum depletion of the condensate $\left[19,83,84\right.$. In superfluid ${ }^{4} \mathrm{He}$, 
for instance, the fraction is no less than about $80-90 \%$ 85, while in cold-atomic condensates it is less than 10 $\%$ because a very weak repulsive interaction can be naturally realized [86; it has been recently observed in the experiment [87] in a box trap. Aside from the validity argument of the approximations used in theoretical studies of Bose polarons, it is interesting to find a missing piece, namely, to examine the open question of how the impurity gives a local feedback to the medium bosons: The impurity inevitably induces a local deformation of the condensate and local quantum fluctuations. Our motivation is to examine such local modifications caused by an impurity localized in a trap potential. In the present paper, therefore, we consider a single Bose polaron in spherically symmetric trap potentials and figure out details of spatial structure of the condensate and Bogoliubov excitations by allowing for the changes induced around the impurity by the impurity-boson interaction.

This paper is organized as follows: In Sec. II we set up our system, write down the effective Hamiltonian in detail, and derive a set of coupled equations under some reasonable approximations. In Sec. III we show numerical results obtained by solving the equations derived in Sec. II. In Sec. IV we end up with summary.

\section{FORMULATION}

In this section, we shall write down a set of equations that describe spatial structure of an impurity and the condensed and excited components of a weakly interacting Bose gas in trap potentials.

\section{A. Effective Hamiltonian}

We consider the zero-temperature system of a single atomic impurity immersed in a dilute atomic Bose gas. The impurity and the gas are trapped in the confinement potentials $V_{\mathrm{I}}(\mathbf{r})$ and $V_{\mathrm{B}}(\mathbf{r})$. Bosons are assumed to interact with each other weakly and repulsively, while the impurity-boson interaction is assumed to be tunable between positive and negative values using the Fano-Feshbach resonance. Such a system can be described by the low energy effective Hamiltonian, $\mathcal{H}=H_{\mathrm{imp}}+H_{\mathrm{B}}+H_{\mathrm{int}}$, that is composed of the part of the trapped single impurity, the part of the trapped boson gas, and the part of the impurity-boson interaction, respectively, i.e.,

$$
\begin{aligned}
H_{\mathrm{imp}} & =\frac{\hat{\mathbf{p}}^{2}}{2 m_{\mathrm{I}}}+\frac{m_{\mathrm{I}} \omega_{\mathrm{I}}^{2}}{2} \hat{\mathbf{q}}^{2}, \\
H_{\mathrm{B}} & =\int_{\mathbf{r}} \hat{\phi}^{\dagger}(\mathbf{r})\left[h_{\mathrm{B}}+\frac{g_{\mathrm{BB}}}{2} \hat{\phi}^{\dagger}(\mathbf{r}) \hat{\phi}(\mathbf{r})-\mu\right] \hat{\phi}(\mathbf{r}), \\
H_{\mathrm{int}} & =g_{\mathrm{IB}} \int_{\mathbf{r}} \hat{\phi}^{\dagger}(\mathbf{r}) \delta^{(3)}(\hat{\mathbf{q}}-\mathbf{r}) \hat{\phi}(\mathbf{r}) .
\end{aligned}
$$

Here, $h_{\mathrm{B}}=-\frac{\hbar^{2} \nabla^{2}}{2 m_{\mathrm{B}}}+V_{\mathrm{B}}(\mathbf{r})$, and we have used the first (second) quantized form for the impurity (bosons). We have introduced the abbreviated notation for the spacial integral: $\int_{\mathbf{r}}=\int d^{3} \mathbf{r}$. For the boson-boson and bosonimpurity interaction, the effective coupling constants and $s$-wave scattering lengths are related by

$$
\begin{aligned}
g_{\mathrm{BB}} & =\frac{4 \pi \hbar^{2}}{m_{\mathrm{B}}} a_{\mathrm{BB}}, \\
g_{\mathrm{IB}} & =\frac{2 \pi \hbar^{2}}{m_{\mathrm{red}}} a_{\mathrm{IB}},
\end{aligned}
$$

where $m_{\text {red }}=m_{\mathrm{B}} m_{\mathrm{I}} /\left(m_{\mathrm{B}}+m_{\mathrm{I}}\right)$.

We aim to figure out the ground state properties of this system at zero temperature. In formulation we first take the expectation of $\mathcal{H}$ with respect to a normalized impurity state $|\mathrm{imp}\rangle$ that is yet to be determined. The resultant expression reads

$$
\begin{aligned}
\mathcal{H}_{\mathrm{B}} & =\langle\mathcal{H}\rangle_{\mathrm{imp}} \\
& =\left\langle H_{\mathrm{imp}}\right\rangle_{\mathrm{imp}}+H_{\mathrm{B}}+g_{\mathrm{IB}} \int_{\mathbf{r}}|\psi(\mathbf{r})|^{2} \hat{\phi}^{\dagger}(\mathbf{r}) \hat{\phi}(\mathbf{r}),
\end{aligned}
$$

where $\psi(\mathbf{r})=\langle\mathbf{r} \mid \mathrm{imp}\rangle$ is the wave function of the impurity, and $\left\langle H_{\mathrm{imp}}\right\rangle_{\mathrm{imp}}=\int_{\mathbf{r}} \psi^{*}(\mathbf{r})\left[-\frac{\hbar^{2} \nabla^{2}}{2 m_{\mathrm{I}}}+V_{\mathrm{I}}(\mathbf{r})\right] \psi(\mathbf{r})$. The $\mathcal{H}_{\mathrm{B}}$ defined above represents an effective Hamiltonian for bosons in the presence of an additional potential due to the impurity wave function.

Now we expand the boson field operator into the condensate and its fluctuations as $\hat{\phi}(\mathbf{r})=\phi(\mathbf{r})+\hat{\varphi}(\mathbf{r})$, where $\phi(\mathbf{r}):=\langle\hat{\phi}(\mathbf{r})\rangle$, with $\langle\cdots\rangle$ defined as the expectation value by vacuum of bosons in the presence of an impurity [88], corresponding to the ground state of the unperturbed Hamiltonian to be specified below. This implies $\langle\hat{\varphi}(\mathbf{r})\rangle=0$. Expanding $\mathcal{H}_{\mathrm{B}}$ with respect to the fluctuations, we obtain

$$
\begin{aligned}
\mathcal{H}^{(0)} & =\left\langle H_{\mathrm{imp}}\right\rangle_{\mathrm{imp}} \\
& +\int_{\mathbf{r}} \phi^{*}\left[h_{\mathrm{B}}+g_{\mathrm{IB}}|\psi|^{2}+\frac{g_{\mathrm{BB}}}{2}|\phi|^{2}-\mu\right] \phi, \\
\mathcal{H}^{(1)} & =\int_{\mathbf{r}} \hat{\varphi}^{\dagger}\left(h_{\mathrm{B}}+g_{\mathrm{IB}}|\psi|^{2}+g_{\mathrm{BB}}|\phi|^{2}-\mu\right) \phi+h . c ., \\
\mathcal{H}^{(2)} & =\frac{1}{2} \int_{\mathbf{r}}\left(\begin{array}{cc}
\hat{\varphi}^{\dagger} & \hat{\varphi}
\end{array}\right)\left(\begin{array}{cc}
\mathcal{L} & \mathcal{M} \\
\mathcal{M}^{*} & \mathcal{L}^{*}
\end{array}\right)\left(\begin{array}{c}
\hat{\varphi} \\
\hat{\varphi}^{\dagger}
\end{array}\right), \\
\mathcal{H}^{(3)} & =g_{\mathrm{BB}} \int_{\mathbf{r}}\left(\phi \hat{\varphi}^{\dagger} \hat{\varphi}^{\dagger} \hat{\varphi}+\phi^{*} \hat{\varphi}^{\dagger} \hat{\varphi} \hat{\varphi}\right) \\
\mathcal{H}^{(4)} & =\frac{g_{\mathrm{BB}}}{2} \int_{\mathbf{r}} \hat{\varphi}^{\dagger} \hat{\varphi}^{\dagger} \hat{\varphi} \hat{\varphi} .
\end{aligned}
$$

where $\mathcal{L}=h_{\mathrm{B}}+g_{\mathrm{IB}}|\psi|^{2}+2 g_{\mathrm{BB}}|\phi|^{2}-\mu$ and $\mathcal{M}=g_{\mathrm{BB}} \phi^{2}$.

Let us now choose $\mathcal{H}^{(1)}+\mathcal{H}^{(2)}$ as the unperturbed Hamiltonian. Then, we find $\mathcal{H}^{(1)}=0$ from $i \hbar \partial_{t}\langle\hat{\varphi}\rangle=$ $\left\langle\left[\hat{\varphi}, \mathcal{H}^{(1)}+\mathcal{H}^{(2)}\right]\right\rangle=0$ in the interaction picture, which leads to the Gross-Pitaevskii equation 89.

$$
\left(h_{\mathrm{B}}+g_{\mathrm{IB}}|\psi|^{2}+g_{\mathrm{BB}}|\phi|^{2}-\mu\right) \phi=0 .
$$

This does not include fluctuation effects explicitly, but only through the impurity's wave function (15). In fact, 
Eq. (12) is equivalent to the stationary condition from $\mathcal{H}^{(0)}$. When higher order fluctuation effects from $\mathcal{H}^{(3)}$ and $\mathcal{H}^{(4)}$ are negligible due to sufficiently weak interactions, i.e., $\mathcal{H}^{(3)}$ and $\mathcal{H}^{(4)}$ terms in the Hamiltonian can be neglected, then the Hamiltonian approximately reads

$$
\mathcal{H}_{\mathrm{B}} \simeq \mathcal{H}^{(0)}+\mathcal{H}^{(2)} .
$$

The ground and excited states for the above approximate Hamiltonian can be determined solely from the diagonalization of $\mathcal{H}^{(2)}$ once the condensation profile is obtained from (12). In the Bogoliubov representation $\hat{\varphi}(\mathbf{r})=\sum_{i}\left[u_{i}(\mathbf{r}) \hat{\alpha}_{i}+v_{i}^{*}(\mathbf{r}) \hat{\alpha}_{i}^{\dagger}\right]$, where the operators $\hat{\alpha}_{i}$ satisfy the canonical commutation relation: $\left[\hat{\alpha}_{i}, \hat{\alpha}_{j}^{\dagger}\right]=\delta_{i j}$, we thus obtain the Bogoliubov-de Gennes (BdG) equations 90, 91,

$$
\left(\begin{array}{cc}
\mathcal{L} & \mathcal{M} \\
-\mathcal{M}^{*} & -\mathcal{L}
\end{array}\right)\left(\begin{array}{l}
u_{i} \\
v_{i}
\end{array}\right)=E_{i}\left(\begin{array}{c}
u_{i} \\
v_{i}
\end{array}\right),
$$

where $E_{i}$ is the eigen-energy of $\mathcal{H}^{(2)}$, i.e., the boson excitation energy. We remark that these excited bosons can be interpreted as phonons, whose bilinear coupling with the impurity is similar to the Fröhlich-type electronphonon coupling in polar semiconductors.

The variational condition with respect to the impurity state $\delta\left\langle\mathcal{H}_{\mathrm{B}}\right\rangle / \delta \psi^{*}=0$ leads to another equation:

$$
\left(h_{\mathrm{I}}+g_{\mathrm{IB}}|\phi|^{2}+g_{\mathrm{IB}}\left\langle\hat{\varphi}^{\dagger} \hat{\varphi}\right\rangle\right) \psi=0,
$$

where the expectation is taken with respect to the bosonic ground state of $\mathcal{H}^{(2)}$, i.e., the Fock vacuum of $\hat{\alpha}_{i}$.

Putting Eqs. 12, 14, and 15 together, we obtain a set of the equations to be solved simultaneously. We note that the chemical potential $\mu$ will be determined from

$$
N_{\mathrm{B}}=\int_{\mathbf{r}}\left\langle\hat{\phi}(\mathbf{r})^{\dagger} \hat{\phi}(\mathbf{r})\right\rangle:=N_{0}+N_{\mathrm{ex}},
$$

where $N_{0}$ is the number of the bosons in the condensate, while $N_{\text {ex }}$ is the number of the bosons in the excited states. At zero temperature, $N_{\text {ex }}$ represents a depletion of the condensate [86], because all the bosons are in the condensate for a non-interacting, impurity-free system. The depletion of the condensate can be described by

$$
N_{\mathrm{ex}}=\sum_{i} \int_{\mathbf{r}}\left|v_{i}(\mathbf{r})\right|^{2},
$$

under the condition that the total number of bosons $N_{B}$ is kept constant by $\mu$.

\section{B. Spherical trap potentials and further approximations}

Hereafter, for theoretical simplicity, we assume that the impurity and the gas are trapped in the respective spherical harmonic-oscillator potentials whose centers coincide: $V_{\mathrm{B}}(\mathbf{r})=\frac{m_{\mathrm{B}} \omega_{\mathrm{B}}^{2}}{2} \mathbf{r}^{2}$ and $V_{\mathrm{I}}(\mathbf{r})=\frac{m_{\mathrm{I}} \omega_{\mathrm{I}}^{2}}{2} \mathbf{r}^{2}$. This theoretical setup is motivated by experiments [12, in which the optical and magneto-optical traps are well described by the harmonic-oscillator potentials.

In this work, we focus on the quantum depletion of the condensate in the presence of an impurity. For simplicity, we ignore the dynamical kickback to the impurity from the medium Bose gas and consider a situation where the impurity is strongly bounded by the spherical harmonic trap potential, i.e., $\hbar \omega_{\ell} \gg\left|g_{\mathrm{IB}} \bar{n}_{\mathrm{B}}\right|$, with $\bar{n}_{\mathrm{B}}$ being an averaged boson density $\bar{n}_{\mathrm{B}}=N_{\mathrm{B}} /\left(\frac{4 \pi}{3} d_{\mathrm{B}}^{3}\right)$ where $d_{\mathrm{B}}=\sqrt{\hbar / m_{\mathrm{B}} \omega_{\mathrm{B}}}$. In this situation, because of the wide energy gap between the ground and other excited states, the wave function of the impurity remains in the ground state. Moreover, the impurity wave function is shrunk by the potential and feels the kickback from the condensate as the constant potential which affects only the energy shift; hence, the kickback term from the condensate in Eq. (15) can be ignored. Therefore, we have

$$
\psi(\mathbf{r}) \simeq\left(\frac{\pi \hbar}{m_{\mathrm{I}} \omega_{\mathrm{I}}}\right)^{-\frac{3}{4}} \exp \left(-\frac{m_{\mathrm{I}} \omega_{\mathrm{I}}}{2 \hbar} r^{2}\right)
$$

We note that this condition is inequivalent to the heavy mass limit of the impurity $m_{\mathrm{I}} \gg m_{\mathrm{B}}$ because the wave function of the impurity is shrunk under this condition but the excitation gap remains unchanged. Since the wave function of the impurity is isotropic, the ground state of the condensate also has an isotropic form,

$$
\phi(\mathbf{r})=\sqrt{\frac{N_{0}}{4 \pi}} \Phi(r),
$$

and the BdG eigenfunctions can be assumed to have a separable form,

$$
\left\{\begin{array}{l}
u_{n_{r} \ell m}(\mathbf{r}) \\
v_{n_{r} \ell m}(\mathbf{r})
\end{array}\right\}=\left\{\begin{array}{l}
\mathcal{U}_{n_{r} \ell}(r) \\
\mathcal{V}_{n_{r} \ell}(r)
\end{array}\right\} Y_{\ell m}\left(\theta_{1}, \theta_{2}\right)
$$

where $\left(n_{r}, \ell, m\right)$ denote the radial, azimuthal, and magnetic quantum number, respectively. Finally, the depletion of the condensate is characterized by

$$
\begin{aligned}
N_{\mathrm{ex}} & =\sum_{n_{r}, \ell} \int_{\mathbf{r}} n_{\mathrm{ex}, n_{r} \ell}(r), \\
n_{\mathrm{ex}, n_{r} \ell}(r) & =\frac{2 \ell+1}{4 \pi}\left|\mathcal{V}_{n_{r} \ell}(r)\right|^{2} .
\end{aligned}
$$

\section{NUMERICAL RESULTS AND DISCUSSION}

Let us now present numerical results for the quantum depletion. To this end, we consider a situation in which a ${ }^{40} \mathrm{~K}$ Fermi impurity is immersed in an ${ }^{87} \mathrm{Rb}$ Bose condensate, i.e., $m_{\mathrm{I}} / m_{\mathrm{B}} \simeq 0.460$. We set the total number of the bosons as $N_{\mathrm{B}}=10^{5}$ and the ratio of the strength of the trap potentials as $\omega_{\mathrm{I}} / \omega_{\mathrm{B}}=5$ with $\omega_{\mathrm{B}}=20 \times 2 \pi \mathrm{Hz}$. For the boson-boson and boson-impurity interactions, we 
take $1 /\left(a_{\mathrm{BB}} \bar{n}_{\mathrm{B}}^{1 / 3}\right)=100$, and $1 /\left(a_{\mathrm{IB}} \bar{n}_{\mathrm{B}}^{1 / 3}\right)= \pm 10$, with

$$
\begin{aligned}
& \bar{n}_{\mathrm{B}}=N_{\mathrm{B}} /\left(\frac{4 \pi}{3} d_{\mathrm{B}}^{3}\right)=1.70 \times 10^{15} \mathrm{~cm}^{-3}, \\
& d_{\mathrm{B}}=\sqrt{\frac{\hbar}{m_{\mathrm{B}} \omega_{\mathrm{B}}}}=2.41 \times 10^{-4} \mathrm{~cm} .
\end{aligned}
$$

Throughout the numerical calculations we keep the number of total bosons $N_{\mathrm{B}}$ fixed by tuning the chemical potential.

\section{A. Condensate and depletion in real space}

We first consider the spatial dependence of the condensate and depletion. Figure 1 shows the radial profile of the order parameter in the absence of the impurity $\Phi^{(0)}(r)$ and the change in the order parameter due to the presence of the impurity, defined by

$$
\delta \Phi(r):=\Phi(r)-\Phi^{(0)}(r) .
$$

Hereafter, we use the superscript (0) that denotes the absence of the impurity. Note that the impurity is localized at the center of the trap potential [92]. When the boson-impurity interaction is repulsive $g_{\mathrm{IB}}>0$ (attractive $g_{\mathrm{IB}}<0$ ), therefore, the condensate feels as if there appeared an additional small bump (dip) at the center, and eventually is repelled a little bit from (pulled toward) the impurity as shown in Fig. 1 .

Correspondingly, Fig. 2 exhibits the radial profile of the density of the quantum depletion, $\sum_{n_{r}, \ell} n_{\mathrm{ex}, n_{r} \ell}(r):=$ $n_{\mathrm{ex}}(r)$, in the absence of the impurity and the change in the density of the quantum depletion due to the presence of the impurity,

$$
\delta n_{\mathrm{ex}}(r):=\sum_{n_{r}, \ell}\left(n_{\mathrm{ex}, n_{r} \ell}(r)-n_{\mathrm{ex}, n_{r} \ell}^{(0)}(r)\right) .
$$

We find that the profiles of the order parameter and the quantum depletion are similar. This result reflects the fact that the quantum depletion at a given position arises from zero-range boson-boson repulsion and thus follows the local density of the condensate. The impurity nearby induces a decrease (increase) in the number of atoms in the condensate by $2.38 \times 10^{-3}\left(2.40 \times 10^{-3}\right)$ and hence leads to a decrease (increase) of the quantum depletion. We remark that in the far region from the impurity, i.e., beyond $r / d_{\mathrm{B}} \sim 1.7$, the change in the condensate and depletion due to the presence of the impurity is tiny, but opposite in sign to that around the impurity.

Finally, we remark on the details of numerical calculations. As pointed out in Ref. [86], the convergence of the sum in Eq. 21) is significantly slow. We truncate the terms whose energy exceeds $1000 / \hbar \omega_{\mathrm{B}}$. Even with this truncation, we can reproduce $90 \%$ of the depletion obtained in the semiclassical approximation [86, which implies that our approach is quantitatively reasonable.


FIG. 1. (Color online) (a) Radial profile of the order parameter $\Phi(r)$ in the absence of an impurity and (b) difference of the radial profile of the order parameter in the presence of an impurity from the result depicted in (a).

\section{B. Depletion vs. quantum numbers}

The $n_{r}$ dependence of the quantum depletion of the condensate is shown for $\ell=0,1,2$ in Fig. 3 . The figure indicates a qualitative difference in the behaviors between $\ell=0$ and $\ell=1,2$ : When $g_{\mathrm{IB}}>0$, the quantum depletion is always suppressed by $\ell=0$ excitations with any $n_{r}$, while it is suppressed (enhanced) by high-lying (low-lying) excitations of $\ell \neq 0$. When $g_{\mathrm{IB}}<0$, the quantum depletion behaves in the opposite direction to when $g_{\text {IB }}>0$.

To understand such behavior we illustrate the BdG eigenfunctions $\mathcal{V}_{n_{r} \ell}(r)$ which correspond to "hole" excitations in the absence of the impurity, and differences in the quantum depletion at each state,

$$
\delta n_{\mathrm{ex}, n_{r} \ell}(r):=n_{\mathrm{ex}, n_{r} \ell}(r)-n_{\mathrm{ex}, n_{r} \ell}^{(0)}(r),
$$

due to the presence of the impurity in Fig. 4. We can see that for given $\ell$ and $n_{r}$, the corresponding change in the density of depletion due to the presence of the impurity is controlled by where the bottom of the dip of the BdG eigenfunction $\mathcal{V}_{n_{r} \ell}(r)$ is located.

It should be noted that the behavior of the "hole" excitation function $\mathcal{V}_{n_{r}, \ell}(r)$ is determined mainly from the effective potential, $V_{\text {eff }, \ell}(r):=V_{\mathrm{B}}(r)+\frac{\hbar^{2} \ell(\ell+1)}{2 r^{2}}+$ $g_{\mathrm{IB}} \frac{\psi^{2}(r)}{4 \pi}+g_{\mathrm{B}} N_{0} \frac{\Phi^{2}(r)}{2 \pi}-\mu$, in the diagonal part of Eq. 14, $\mathcal{L}_{\ell}=-\frac{\hbar^{2}}{2 m_{\mathrm{B}}}\left(\frac{d^{2}}{d r^{2}}+\frac{2}{r} \frac{d}{d r}\right)+V_{\mathrm{eff}, \ell}(r)$. We show the differ- 

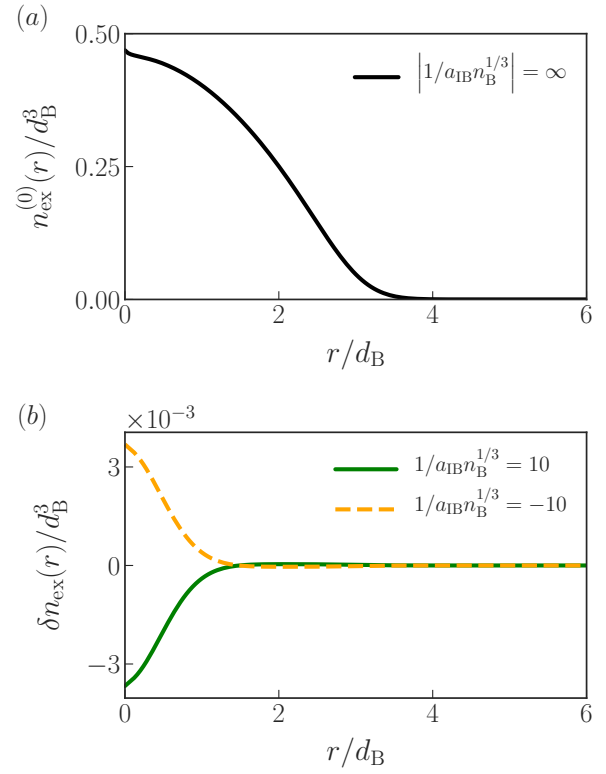

FIG. 2. (Color online) Same as Fig. 1 for the density of depletion, $\sum_{n_{r}, \ell} n_{\mathrm{ex}, n_{r} \ell}(r)$.

ence of the effective potential in the presence of an impurity from that in its absence: $\delta V_{\mathrm{eff}, \ell}(r):=V_{\mathrm{eff}, \ell}(r)-V_{\mathrm{eff}, \ell}^{(0)}$ in Fig. 5 .

Figure 5 shows that the $\delta V_{\text {eff }, \ell}(r)$ directly reflects the difference of the radial profile of the condensate (see Fig. 1(b) $)$ and changes the sign at $r / d_{\mathrm{B}} \sim 1.7$. When the peak of $\mathcal{V}_{n_{r}, \ell}(r)$ comes near this point, the sign of the quantum depletion changes. In the case of $\ell=0$ in which no centrifugal potential occurs, the dip bottom of $\mathcal{V}_{n_{r} \ell}(r)$ appears at the center of the effective potential, as shown in Fig. 4, and the presence of the impurity gives a small isotropic change to the potential around the center, which thus leads to negative or positive $\delta n_{\mathrm{ex}, n_{r} 0}(r)$ therein. On the other hand, when $\ell \neq 0$, the presence of the centrifugal potential allows the dip bottom of $\mathcal{V}_{n_{r} \ell \neq 0}(r)$ to move away from $r=0$ and, for sufficiently small $n_{r}$ (corresponding to sufficiently high "hole" excitation level $-E_{i}$ ), to enter the regime where the impurity-induced change in the density of the condensate is opposite in sign, while $\mathcal{V}_{n_{r} \ell \neq 0}(0)=0$ is required for regularization. Consequently, the change in the depletion for $\ell \neq 0$ appears inevitably away from the center but within the influence of the impurity's wave function, which can be summarized in terms of real space densities at $z=0$ in Figs. 6 and 7 . For these reasons, the depletion of the condensate depends strongly on the excitation energy as well as the $\ell$ value.

We note that the depletion for $\ell=0$ is relatively small in comparison with that for $\ell=1,2$. Since the integration (21) includes the Jacobian $\propto r^{2}$, the contributions from the amplitude of $\mathcal{V}_{n_{r}, \ell=0}(r)$ dipped around $r=0$ are suppressed, while those from the amplitudes of $\mathcal{V}_{n_{r}, \ell \neq 0}(r)$ around the dip bottoms remain unsuppressed. This is why the depletion for $\ell=0$ is smaller than $\ell=1,2$. This situation is the same as the problems of quantum mechanics in harmonic oscillators.

\section{SUMMARY}

We have studied the quantum depletion of the condensate in the trapped Bose-polaron system and found that the net change of the depletion, $\delta N_{\mathrm{ex}}=$ $\sum_{n_{r}, \ell} \int_{\mathbf{r}} \delta n_{\mathrm{ex}, n_{r} \ell}(r)$, induced by the impurity can be negative (positive) for the repulsive (attractive) impurityboson interaction. These qualitative results can be understood as follows: 1) We have solved a set of coupled equations for the condensate, the impurity's wave function, and Bogoliubov excitations. 2) Among them, the first two are apparently coupled with each other, but the condensate alone is affected by the other because we have ignored the kickback effect on the impurity by assuming that the impurity is tightly bounded in the trap. 3) Then, the resultant condensate, together with the impurity's wave function, gives a small dip (bump) to the effective potential in the BdG equation (see Fig 5), which determines the spectra of excitations, i.e., the depletion.

The negative change of the depletion that we have found in the case of the repulsive impurity-boson interaction in this study seems counter intuitive, since in general the impurity-boson interaction, no matter whether repulsive or attractive, disturbs the condensation in uniform systems. Thus, we consider this result as a specific feature in trapped systems. Moreover, this result does not depend on the shape of trap potentials and can be observed, for example, in a box trap potential.

Finally, we give a brief comment on possible experimental observations of the results found in this study. Although the single-impurity-induced change in the number of depletion is minuscule, i.e., $\left|\delta N_{\mathrm{ex}}\right| \sim 10^{-3}$ for weak coupling considered here, we expect that such change in the number of depletion is detectable for a dilute impurity gas even in weak coupling and more easily in strong coupling. For instance, in-situ experiments [93 98 ] could be used to obtain images of bosonic excitations, while the photoabsorption spectroscopy by lasers 99 , 100] could select the azimuthal quantum number $\ell$ in the excitation number $N_{\mathrm{ex}, n_{r} \ell}$. Nevertheless, these experimental observations seem challenging because of smallness of the depletion. To enhance the impurity-induced change in the quantum depletion, we propose a method that uses bosonic impurities. With this setting, the amplitude of the impurity wave function is increased; therefore, the impurity-induced change in the number of the quantum depletion can be enhanced. For studies of such situations, we will progressively extend the framework of our theory to deal with the system with many impurities , incorporating the many-body effects of impurities, e.g., 

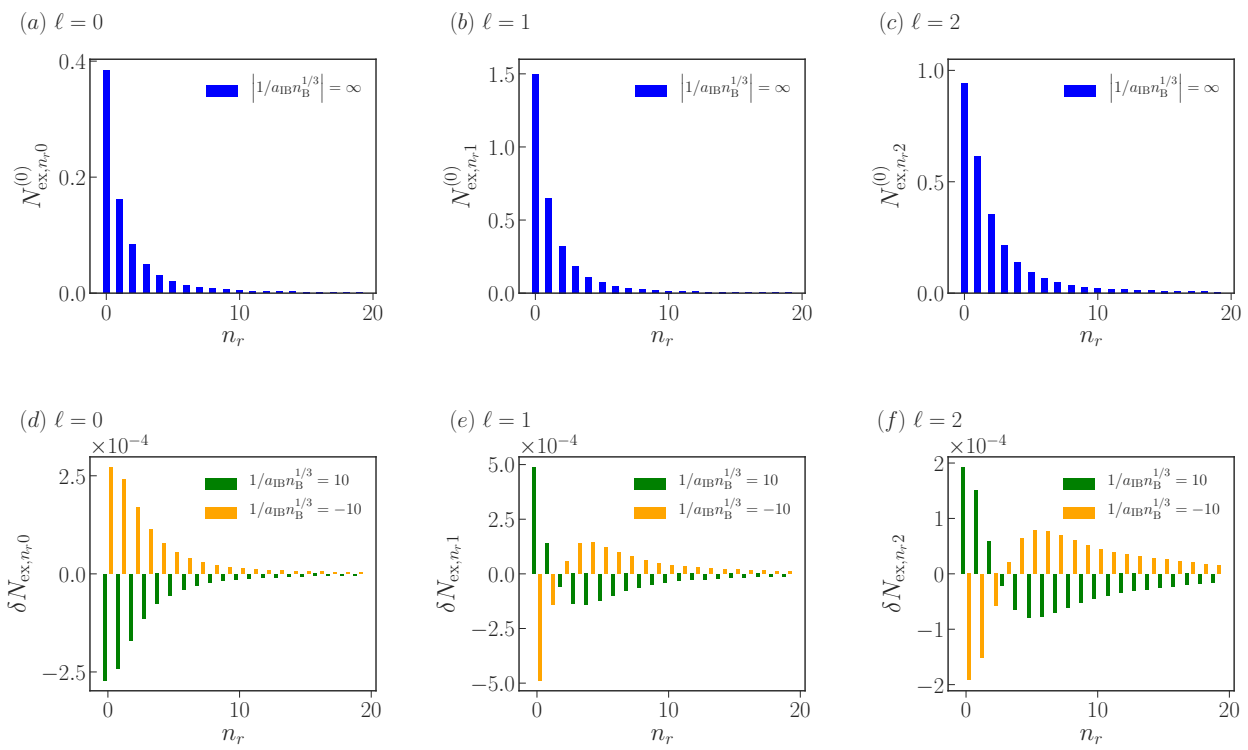

FIG. 3. (Color online) The number of the quantum depletion, $N_{\mathrm{ex}, n_{r} \ell}=\int_{\mathrm{r}} n_{\mathrm{ex}, n_{r} \ell}(r)$, obtained as function of $n_{r}$ for $\ell=0,1,2$. The impurity-free cases are depicted in (a)-(c), while the impurity-induced changes are in (d) $-(\mathrm{f})$.

the effective impurity-impurity interactions mediated by medium fluctuations [18, 82.

For another direction of research, we will further develop the present method to look into zero-mode physics 101 103, few-body physics, self-localization, and so on.

\section{ACKNOWLEDGMENTS}

We are grateful to the RIKEN symposium Thermal field theory and its applications 2018 supported by
iTHEMS, where the present work was initiated. This work was supported in part by Grants-in-Aid for Scientific Research through Grant Nos. 17K05445, 18K03501, 18H05406, and 18H01211, provided by JSPS.
[1] L. D. Landau, Phys. Z. Sowjetunion 3, 664 (1933); L. Landau and S. Pekar, J. Exptl. Theor. Phys. 18, 419 (1948); S. Pekar, J. Exptl. Theor. Phys. 19, 796 (1949).

[2] H. Fröhlich, Theory of Dielectrics, (Clarendon Press, Oxford, 1949); H. Fröhlich, H. Pelzer, and S. Zienau, Phil. Mag. 41, 221 (1950); H. Fröhlich, Adv. Phys. 3, 325 (1954).

[3] T. D. Lee, F. E. Low, and D. Pines, Phys. Rev. 90, No.2, 297-302 (1953).

[4] Gerald D. Mahan, Many-Particle Physics (Springer, 1990).

[5] See, for instance, edited by E. K. H. Salje, A. S. Alexandrov, and W. Y. Liang, Polarons and Bipolarons in High-Tc Superconductors and Related Materials Cambridge University Press; Revised (Cambridge, 2005)

[6] A. S. Alexandrov, J. T. Devreese, Advances in Polaron Physics, Springer Series in Solid-State Sciences Vol. 159, (Springer, 2009).
[7] J. Catani, G. Lamporesi, D. Naik, M. Gring, M. Inguscio, F. Minardi, A. Kantian, and T. Giamarchi, Phys. Rev. A 85, 023623 (2012).

[8] R. Scelle, T. Rentrop, A. Trautmann, T. Schuster, and M. K. Oberthaler, Phys. Rev. Lett. 111, 070401 (2013).

[9] M. Hohmann, F. Kindermann, B. Gänger, T. Lausch, D. Mayer, F. Schmidt and A. Widera, EPJ Quantum Technology 2:23, (2015)

[10] E. Compagno, G. De Chiara, D. G. Angelakis, and G. M. Palma, Scientific Reports vol. 7, 2355 (2017).

[11] N. B. Jørgensen, L. Wacker, K. T. Skalmstang, M. M. Parish, J. Levinsen, R. S. Christensen, G. M. Bruun, J. J. Arlt, Phys. Rev. Lett. 117, 055302 (2016).

[12] M. -G. Hu, M. J. Van de Graaff, D. Kedar, J. P. Corson, E. A. Cornell, and D. S. Jin, Phys. Rev. Lett. 117, 055301 (2016).

[13] T. Rentrop, A. Trautmann, F. A. Olivares, F. Jendrzejewski, A. Komnik, and M. K. Oberthaler, Phys. Rev. X 


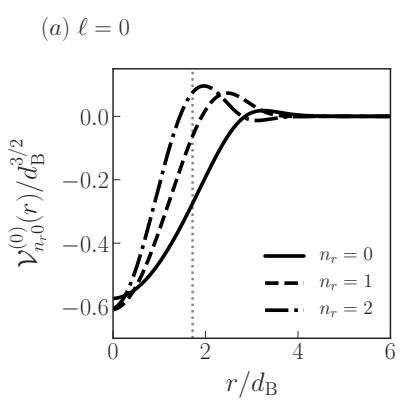

(d) $\ell=0$

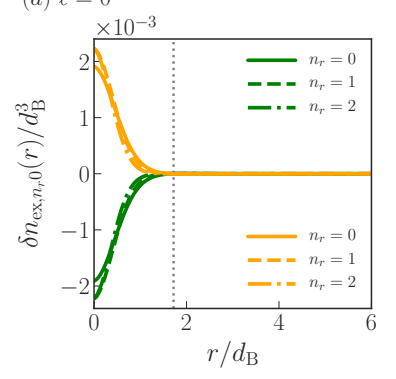

(b) $\ell=1$

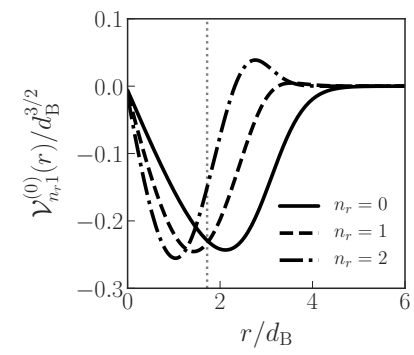

(e) $\ell=1$

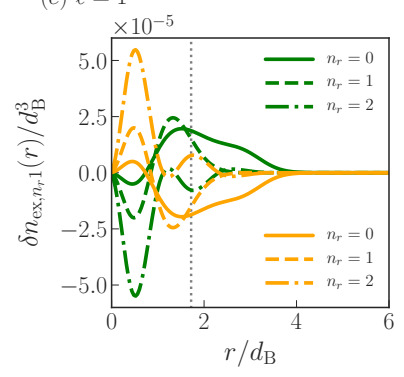

(c) $\ell=2$

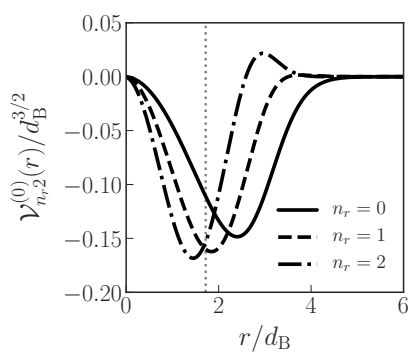

(f) $\ell=2$

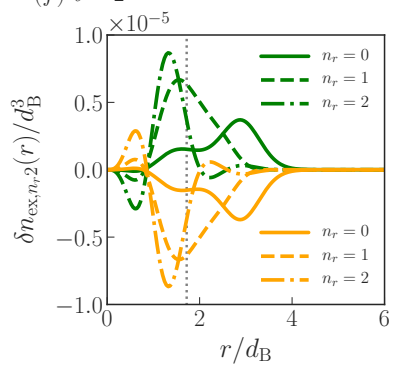

FIG. 4. (Color online) (a)-(c) Radial profile of the BdG eigenfunctions $\mathcal{V}_{n_{r} \ell}(r)$ in the absence of the impurity and (d)-(f) the corresponding change in the density of the quantum depletion due to the presence of the impurity. In (d)-(f), the green and the orange lines denote the $1 / a_{\mathrm{IB}} n_{\mathrm{B}}^{1 / 3}=+10$ and -10 , respectively. The vertical dotted lines are drawn in the same way as in Fig. 5 .

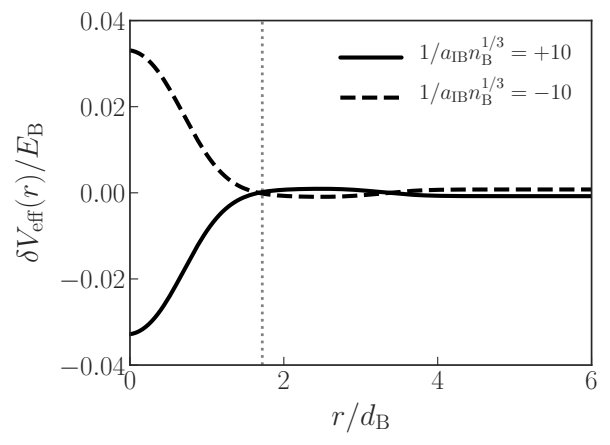

FIG. 5. (Color online) Difference of the radial profile of the effective potential nondimensionalized by $E_{\mathrm{B}}=\frac{\hbar \omega_{\mathrm{B}}}{2}$. The vertical dotted line denotes the point where the sign of $\delta V_{\text {eff }}$ changes.

6, 041041 (2016).

[14] A. Schirotzek, C. -H. Wu, A. Sommer, and M. W. Zwierlein, Phys. Rev. Lett. 102, 230402 (2009).

[15] B. Fröhlich, M. Feld, E. Vogt, M. Koschorreck, W. Zwerger, and M. Köhl, Phys. Rev. Lett. 106, 105301 (2011).

[16] C. Kohstall, M. Zaccanti, M. Jag, A. Trenkwalder, P. Massignan, G. M. Bruun, F. Schreck, and R. Grimm, Nature 485, 615-618 (2012).
[17] F. Scazza, G. Valtolina, P. Massignan, A. Recati, A. Amico, A. Burchianti, C. Fort, M. Inguscio, M. Zaccanti, and G. Roati, Phys. Rev. Lett. 118, 083602 (2017).

[18] M. Cetina, M. Jag, R. S. Lous, I. Fritsche, J. T. M. Walraven, R. Grimm, J. Levinsen, M. M. Parish, R. Schmidt, M. Knap, and E. Demler, Science 354, 96 (2016).

[19] See, for instance, C. J. Pethick and H. Smith, BoseEinstein Condensation in Dilute Gases (Cambridge University Press, Cambridge, 2008).

[20] J. Tempere, W. Casteels, M. K. Oberthaler, S. Knoop, E. Timmermans, and J. T. Devreese, Phys. Rev. B 80, 184504 (2009).

[21] W. Li and S. Das Sarma, Phys. Rev. A 90, 013618 (2014).

[22] A. Shashi, F. Grusdt, D. A. Abanin, and E. Demler, Phys. Rev. A 89, 053617 (2014).

[23] A. S. Dehkharghani, A. G. Volosniev, and N. T. Zinner, Phys. Rev. A 92, 031601(R) (2015).

[24] R. S. Christensen, J. Levinsen, and G. M. Bruun, Phys. Rev. Lett. 115, 160401 (2015)

[25] J. Vlietinck, W. Casteels, K. Van Houcke, J. Tempere, J. Ryckebusch, and J. T. Devreese, New J. Phys. 17, 033023 (2015).

[26] F. Grusdt, Y. E. Shchadilova, A. N. Rubtsov, and E. Demler, Sci. Rep. 5, 12124 (2015).

[27] F. Grusdt and M. Fleischhauer, Phys. Rev. Lett. 116, 053602 (2016).

[28] Y. E. Shchadilova, F. Grusdt, A. N. Rubtsov, and E. Demler, Phys. Rev. A 93, 043606 (2016) 

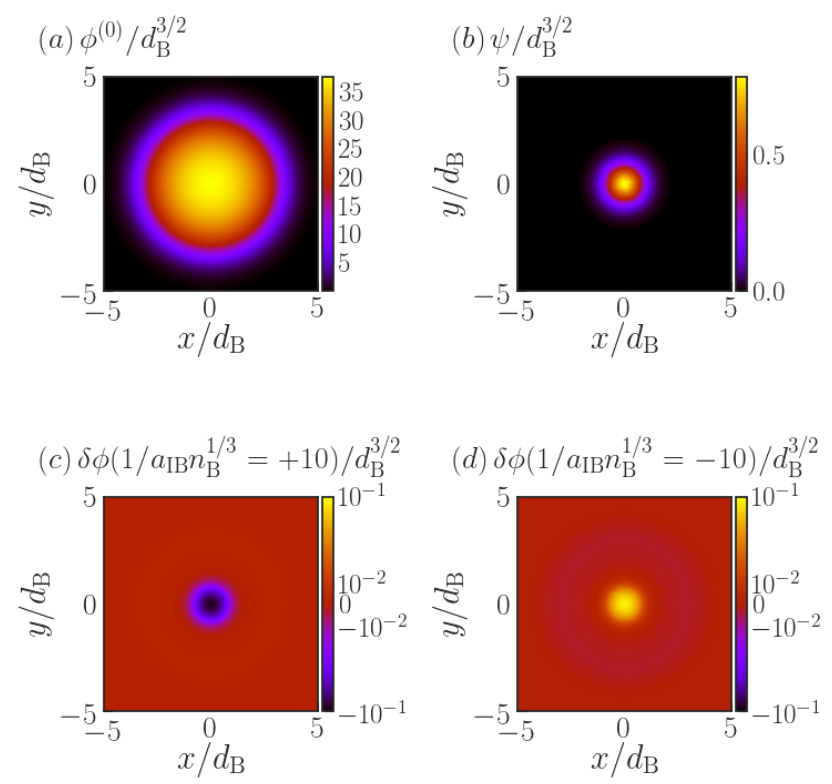

FIG. 6. (Color online) Real space profiles at $z=0$ of (a) the order parameter $\phi_{\mathrm{B}}(x, y, z=0)$ in the absence of the impurity, (b) the impurity's wave function $\psi_{\mathrm{I}}(x, y, z=0)$, and the differences of the order parameter $\delta \phi_{\mathrm{B}}(x, y, z=0)$ in the presence of the impurity with (c) repulsive and (d) attractive boson-impurity interactions.

[29] A. A. Blinova, M. G. Boshier, E. Timmermans, Phys. Rev. A 88, 053610 (2013).

[30] M. Drescher, M. Salmhofer, T. Enss, Phys. Rev. A 99, 023601 (2019).

[31] F. Chevy, Phys. Rev. A 74, 063628 (2006);

[32] Unitary polarized Fermi gases, p. 607 in Ultra-Cold Fermi Gases, Eds. M. Inguscio, W. Ketterle, C. Salomon, (IOS Press, Amsterdam, 2007).

[33] P. Massignan, G. M. Bruun, and H. T. C. Stoof Phys. Rev. A 78, 031602(R) (2008).

[34] R. Schmidt and T. Enss, Phys. Rev. A 83, 063620 (2011).

[35] Attractive and repulsive Fermi polarons in two dimensions M. Koschorreck, D. Pertot, E. Vogt, B. Fröhlich, M. Feld, and M. Köhl, Nature 485, 619 (2012).

[36] R. Schmidt, T. Enss, V. Pietilä, and E. Demler, Phys. Rev. A 85, 021602(R) (2012).

[37] J. Vlietinck, J. Ryckebusch, and K. Van Houcke, Phys. Rev. B 87, 115133 (2013).

[38] C. Trefzger and Y. Castin, Europhysics Letters 104, 50005 (2013).

[39] C. Trefzger and Y. Castin, Phys. Rev. A 90, 033619 (2014).

[40] P. Massignan, M. Zaccanti, and G. M. Bruun, Reports on Progress in Physics, 77, 034401, (2014).

[41] Z. Lan and C. Lobo, Phys. Rev. A 92, 053605 (2015)

[42] W. Yi and X. Cui Phys. Rev. A 92, 013620 (2015).

[43] K. Kamikado, T. Kanazawa, and S. Uchino, Phys. Rev. A 95, 013612 (2017).
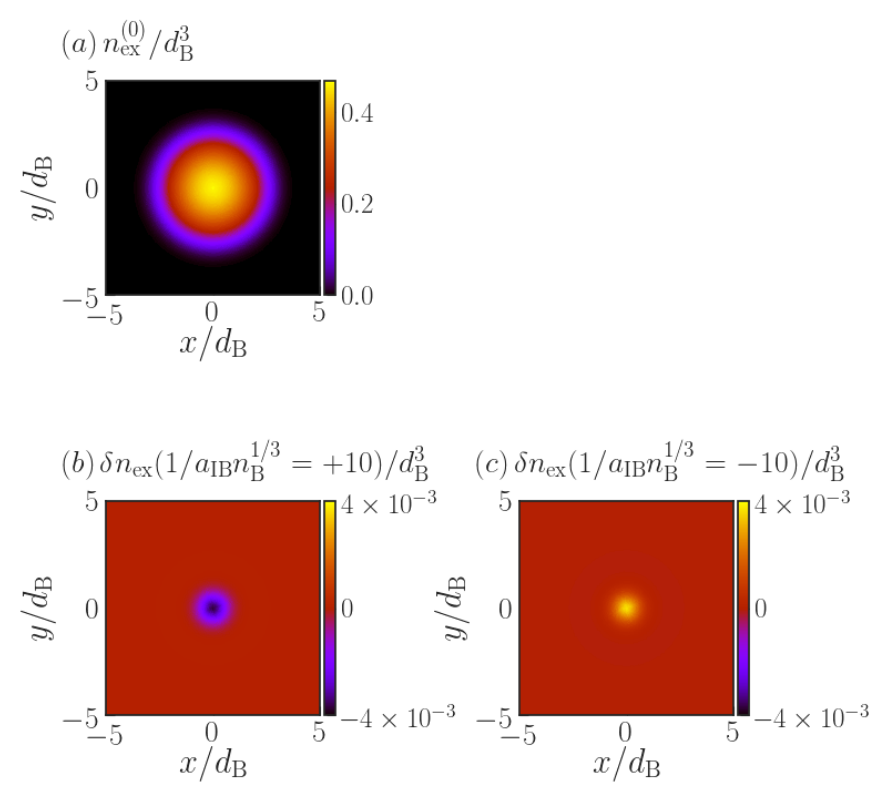

FIG. 7. (Color online) Real space profiles at $z=0$ of (a) the density of depletion in the absence of the impurity $n_{\mathrm{ex}}(x, y, z=0)$ and the differences of the density of depletion $\delta n_{\mathrm{ex}}(x, y, z=0)$ in the presence of the impurity with (b) repulsive and (c) attractive boson-impurity interactions.

[44] B. Kain and H. Y. Ling, Phys. Rev. A 96, 033627 (2017)

[45] F. M. Cucchietti and E. Timmermans, Phys. Rev. Lett. 96, 210401 (2006).

[46] K. Sacha and E. Timmermans, Phys. Rev. A 73, 063604 (2006).

[47] R. M. Kalas, and D. Blume, Phys. Rev. A 73, 043608 (2006).

[48] M. Bruderer, W. Bao, D. Jaksch, EuroPhys. Lett. 82, 30004 (2008).

[49] A. Boudjemaa, Phys. Rev. A 90, 013628 (2014).

[50] W. Casteels, T. Van Cauteren, J. Tempere, and J. T. Devreese, Laser Physics 21, 8, pp 1480-1485 (2011).

[51] S. P. Rath and R. Schmidt, Phys. Rev. A, 88, 053632 (2013)

[52] L. A. Peña Ardila and S. Giorgini, Phys. Rev. A 92, 033612 (2015).

[53] R. Schmidt, M. Knap, D. A. Ivanov, J.-S. You, M. Cetina, E. Demler, Rep. Prog. Phys. 81, 024401 (2018).

[54] L. A. Peña Ardila, N. B. Jørgensen, T. Pohl, S. Giorgini, G. M. Bruun, and J. J. Arlt, arXiv:1812.04609.

[55] J. Levinsen, M. M. Parish, and G. M. Bruun, Phys. Rev. Lett. 115, 125302 (2015).

[56] J. Levinsen, P. Massignan, S. Endo, M. M. Parish, J. Phys. B: At. Mol. Opt. Phys. 50, 072001 (2017).

[57] Mingyuan Sun, Hui Zhai, and Xiaoling Cui, Phys. Rev. Lett. bf 119, 013401 (2017).

[58] P. Naidon, J. Phys. Soc. Jpn. 87, 043002 (2018) 
[59] Y. E. Shchadilova, R. Schmidt, F. Grusdt, and E. Demler, Phys. Rev. Lett. 117, 113002 (2016).

[60] M. M. Parish and J. Levinsen, Phys. Rev. B 94, 184303 (2016).

[61] F. Grusdt, K. Seetharam, Y. Shchadilova, and E. Demler, Phys. Rev. A 97, 033612 (2018).

[62] Y. Ashida, R. Schmidt, L. Tarruell, and E. Demler Phys. Rev. B 97, 060302(R) (2018).

[63] Aniello Lampo, Christos Charalambous, Miguel Ángel Garcia-March, and Maciej Lewenstein, Phys. Rev. A - 98, 063630 (2018).

[64] K. K. Nielsen, L. A. Peña Ardila, G. M. Bruun, and T. Pohl, New J. Phys. 21, 043014 (2019).

[65] S. I. Mistakidis, G. C. Katsimiga, G. M. Koutentakis, Th. Busch, P. Schmelcher, Phys. Rev. Lett. 122, 183001 (2019).

[66] J. Levinsen, P. Massignan, F. Chevy, and C. Lobo, Phys. Rev. Lett. 109, 075302 (2012).

[67] B. Kain and H. Y. Ling, Phys. Rev. A 89, 023612 (2014).

[68] Ground-state properties of Dipolar Bose polarons, L. A. Peña Ardila and T. Pohl, J. Phys. B: At. Mol. Opt. Phys. 52, 015004 (2019).

[69] R. Schmidt and M. Lemeshko, Phys. Rev. Lett. 114, 203001 (2015).

[70] R. Schmidt and M. Lemeshko, Phys. Rev. X 6, 011012 (2016).

[71] E. Yakaboylu, B. Midya, A. Deuchert, N. Leopold, and M. Lemeshko Phys. Rev. B 98, 224506 (2018).

[72] X. Li, G. Bighin, E. Yakaboylu, M. Lemeshko, Molecular Physics,1-8 (2019):

[73] J. Levinsen, M. M. Parish, R. S. Christensen, J. J. Arlt, and G. M. Bruun, Phys. Rev.-A 96, 063622 (2017).

[74] N.-E. Guenther, P. Massignan, M. Lewenstein, and G. M. Bruun, Phys. Rev. Lett.120, 050405 (2018).

[75] Boiling a Unitary Fermi Liquid, Z. Yan, P. B. Patel, B. Mukherjee, R. J. Fletcher, J. Struck, and M. W. Zwierlein, Phys. Rev. Lett. 122, 093401 (2019).

[76] H. Tajima and S. Uchino, New J. Phys. 20, 073048 (2018).

[77] Thermal crossover, transition, and coexistence in Fermi polaronic spectroscopies, H. Tajima and S. Uchino, arXiv:1812.05889

[78] Z. Z. Yan, Y. Ni, C. Robens, M. W. Zwierlein, arXiv:1904.02685.

[79] E. Nakano, H. Yabu, and K. Iida, Phys. Rev. A 95, 023626 (2017).

[80] K. Watanabe, E. Nakano, and H. Yabu, Phys. Rev. A 99, 033624 (2019).

[81] M. Bruderer, Alexander Klein, Stephen R. Clark, and Dieter Jaksch, Phys. Rev. A 76, 011605(R) (2007); New J. Phys. 10, 033015 (2008).
[82] E. Nakano and H. Yabu, Phys. Rev. B 93, 205144 (2016).

[83] F. Dalfovo, S. Giorgini, L. P. Pitaevskii, and S. Stringari, Rev. Mod. Phys. 71, 463 (1999).

[84] L. Pitaevskii and S. Stringari, Bose-Einstein Condensation (Oxford, New York, 2003).

[85] See, for instance, A. J. Leggett, Quantum Liquids (Oxford, New York, 2006).

[86] F. Dalfovo, S. Giorgini, M. Guilleumas, L. Pitaevskii, and S. Stringari, Phys. Rev. A 56, 3840 (1997).

[87] R. Lopes, C. Eigen, N. Navon, D. Clément, R. P. Smith, Z. Hadzibabic, Phys. Rev. Lett. 119, 190404 (2017).

[88] The definition of the expectation depends on the choice of the unperturbed Hamiltonian and the approximation scheme.

[89] E. P. Gross, Nuovo Cimento 20, 454 (1961); J. Math. Phys. 4, 195 (1963); L. P. Pitaevskii, Zh. Eksp. Teor. Fiz. 40, 646 (1961).

[90] N. N. Bogoliubov, J. Phys. (Moscow) 11, 32 (1947).

[91] P. G. de Gennes, Superconductivity of Metals and Alloys (Benjamin, New York, 1966).

[92] We do not discuss here the self-localization of impurities 45, 46. The weak boson-impurity coupling constant used here keeps the system from a possible Bose collapse expected for strong attractive couplings in trapped systems 47, 48.

[93] Y. -I. Shin, C. H. Schunck, A. Schirotzek, and W. Ketterle, Nature 451, 689-693 (2008).

[94] W. S. Bakr, J. I. Gillen, A. Peng, S. Fölling, and M. Greiner, Nature 462, 74-77 (2009).

[95] J. F. Sherson, C. Weitenberg, M. Endres, M. Cheneau, I. Bloch, and S. Kuhr, Nature 467, 68-72 (2010).

[96] M. Horikoshi, S. Nakajima, M. Ueda, and T. Mukaiyama, Science 327, 442 (2010).

[97] M. J. H. Ku, A. T. Sommer, L. W. Cheuk, and M. W. Zwierlein, Science 335, 563 (2012).

[98] A. Alberti, C. Robens, W. Alt, S. Brakhane, M. Karski, R. Reimann, A. Widera, and D. Meschede, New J. Phys. 18, 053010 (2016).

[99] M. R. Matthews, B. P. Anderson, P. C. Haljan, D. S. Hall, C. E. Wieman, and E. A. Cornell, Phys. Rev. Lett. 83, 2498 (1999).

[100] K. W. Madison, F. Chevy, W. Wohlleben, and J. Dalibard, Phys. Rev. Lett. 84, 806 (2000); J. R. AboShaeer, C. Raman, J. M. Vogels, W. Ketterle, Science 292, 476 (2001).

[101] Y. Nakamura, J. Takahashi, and Y. Yamanaka, Phys. Rev. A 89013613 (2014).

[102] Y. Nakamura, T. Kawaguchi, Y. Torii, and Y. Yamanaka, Annals of Physics 376, 484 (2017).

[103] J. Takahashi, Y. Nakamura, and Y. Yamanaka, Phys. Rev. A 92, 023627 (2015). 